\title{
Challenges of Hypertension Prevention in the Social Determinants of Stress: A Qualitative Study
}

Fateme Arabi Basharic ${ }^{1}$, Ali Janati ${ }^{2}$, Rahim Khodayari-Zarnaq ${ }^{3}$, Mohammad Zakaria Pezeshki ${ }^{4}$, Noora Rafiee ${ }^{5}$ and Masoumeh Gholizadeh6,*

${ }^{1}$ Department of Management and Health Policy, School of Management and Medical Informatics, Tabriz University of Medical Sciences, Tabriz, Iran

2Department of Management and Health Policy, School of Health Services Management and Medical Informatics, Tabriz University of Medical Sciences, Tabriz, Iran

${ }^{3}$ Health Services Management Research Centre, Health Management and Safety Promotion Research Institute, Tabriz University of Medical Sciences, Tabriz, Iran

${ }^{4}$ Social Determinants of Health Research Centre, Health Management and Safety Promotion Research Institute, Department of Community and Family Medicine, Tabriz Medical School, Tabriz University of Medical Sciences, Tabriz, Iran

${ }^{5}$ Student Research Committee, Department of Health Management, Policy and Economics, School of Management and Medical Informatics, Kerman University of Medical Sciences, Kerman, Iran

${ }^{6}$ Department of Management and Health Policy, School of Health Services Management and Medical Informatics, Tabriz University of Medical Sciences, Tabriz, Iran

* Corresponding author: Masoumeh Gholizadeh, Department of Management and Health Policy, School of Health Services Management and Medical Informatics, Tabriz University of Medical Sciences, Daneshgah Ave., Tabriz, Iran. Tel: +989143068619; Email: mgholizadehm@gmail.com

Received 2021 March 11; Revised 2021 July 05; Accepted 2021 September 20.

\begin{abstract}
Background: Human life is tied with the stress caused by economic, political, social, and cultural problems, which may lead to physical and mental diseases. In such stressful conditions, people make lifestyle changes that put them at high risk for developing hypertension. Objectives: Accordingly, this study investigated the prevention and management of hypertension as a major public health challenge in Iran. Methods: Semi-structured interviews were conducted with managers, health policymakers, social medicine specialists, and faculty members. All interviews were recorded, transcribed verbatim, and analyzed using thematic content analysis. The MAXQDA18 software was applied to facilitate the organization of codes and themes.

Results: Interviews were conducted with 17 participants. The analyses resulted in five themes including, educational policies, cultural policies, urban transport policies, organizational policies, and economic policies. A total of 13 subthemes were also extracted from the data. Conclusion: Findings have indicated the stressors factors, stemming from macro-policies and mismanagement of government. To reduce the burden of hypertension and improve people's living conditions, health-oriented policies should be designed and implemented in all sectors.
\end{abstract}

Keywords: Challenges, Determinant, High blood pressure, Solutions, Stress

\section{Background}

Hypertension is one of the most public health problems (1) and an effective risk factor for cardiovascular diseases (CVDs) (2). The increasing prevalence of hypertension has affected nations, especially developing countries. Many common risk factors, including old age, family history of the disease, overweight and obesity, sedentary lifestyle, and smoking were reported for hypertension (3). Social determinants of health are also considered important risk factors of hypertension $(4,5)$. "Social determinants of health" are defined as "the circumstances in which people are born, grow, live, work, and age, and the systems put in place to deal with illness" $(4,6)$. Considering the recent transformations to our social organization caused by rapid globalization, as well as social and cultural changes, individuals have been subjected to significant psychosocial stress (7). In this regard, urbanization, as a process of expansion in cities due to industries and the economy, has affected various dimensions of life and caused the occurrence of CVD risk factors, such as hypertension (8).

The people's lifestyle and social interactions have changed dramatically as a result of agricultural and industrial developments. In adaptation to the new physical and social environments, individuals are faced with a substantial amount of psychosocial stress induced by behavioral and hormonal responses to stressful experiences (3). Based on the literature, psychosocial factors, such as stress, depression (symptoms), and anxiety can lead to the prevalence of hypertension (1).

According to the recent findings, the pathophysiology of hypertension and CVD is significantly affected by chronic exposure to psychological stressors. Stress influences the pathogenesis of physical disease by affecting biological processes directly or behavioral patterns indirectly. In the case that stress exceeds the adaptive capacity, some maladaptive processes will be obtained that affect the individuals' cardiovascular health adversely (6). According to the prior evidence, perceived stress elevated blood pressure and developed hypertension and CVD (9). Stress has always occupied a place among the important risk factors of hypertension, such as obesity, sedentary lifestyle, and smoking. Modern lifestyle is occupied with mental or psychosocial stress, including 
pressure, troubles, disappointments, worries, and hurries, which can be considered the main risk factors of hypertension (10).

Considering the rapid growth of the modern lifestyle, as well as various social, population, and economic changes in Iran through the past decades, the CVD risk factors had an increasing prevalence (10). Among risk factors of cardiovascular diseases, hypertension is still the main factor for $20 \%-50 \%$ of deaths (11). In Iran, cardiovascular diseases are the most leading cause of death (12). Past studies have mentioned controlling high blood pressure through medication and diet $(13,14)$; however, social determinants of health, such as stress, have been less discussed. Accordingly, it is necessary to have complete information about the social causes of disease and challenges in the community.

\section{Objectives}

The present study was conducted to determine and investigate the challenges of preventing hypertension in social determinants of stress. Our findings can help policymakers, authorities, and planners to modify the existing policies appropriately.

\section{Methods}

This qualitative study was conducted using individual and semi-structured interviews. To collect the study data, interviews were undertaken with managers, health policymakers, social medicine specialists, and faculty members. The participants were asked to provide their viewpoints about the challenges of performing administrative policies and conducting interventions to prevent hypertension.

\subsection{Participants}

The study participants included 17 experts recruited from the Ministry of Health and two universities that were consisted of managers $(n=7)$, health policymakers $(n=2)$, social medicine specialists $(n=4)$, and faculty members $(n=4)$ (Table 1). Participants with at least five years of work experience in the health system were eligible to participate in the study. The interviews lasted from 12 to $69 \mathrm{~min}$ with a mean duration of about $35 \mathrm{~min}$.

\subsection{Semi-structured interviews}

To extract the most relevant questions for the interview, a rapid review of the recent articles was initially conducted (last five years), and several questions were extracted. Subsequently, with the cooperation of the research team, the most appropriate questions were selected and finalized (three questions).

\subsection{Recruitment}

In total, 11 males and 6 females were selected using the purposive sampling method. The participants were provided with some information about the study goals and process. Following that, they were required to sign the informed consent forms to enter the study. Verbal consent was also confirmed before the interview. A mutually convenient interview time and method (telephone or face-to-face) were arranged. As a result, 18 people expressed their interest to participate in the study; however, written consent was received from 17 individuals. The number of participants interviewed was based on the number needed to achieve theoretical data saturation (15). In other words, the research team decided about the novelty of information achieved after each interview. The recruitment ceased at the $17^{\text {th }}$ interview since no new information was achieved upon the $16^{\text {th }}$ and $17^{\text {th }}$ interviews.

\subsection{Data collection}

The research data were collected from January to May 2020, and each participant was interviewed individually. At the individuals' desire, some interviews were conducted face-to-face $(n=8)$ at the participant's place of work, and some others were conducted via telephone $(n=9)$. All Interviews were recorded using a digital sound recording device and transcribed verbatim to word files. Interviews involved three open questions as follows:

In your opinion, what factors cause the most stress in society? How would you describe the effect of stress on hypertension?

In your opinion, what are the challenges related to the prevention of high blood pressure and stress in society?

What strategies and interventions are effective in reducing stress and preventing high blood pressure?

\subsection{Data Analysis}

Thematic content analysis was applied to analyze the data. Explicit rules of coding were employed to compress the texts into replicable fewer content categories systematically. In content analysis, 17 transcribed interviews were analyzed by two different members of the research team. The MAXQDA18 software was run to organize codes and themes. Themes and sub-themes were derived through an inductive process and developed to facilitate a better understanding of the research subject. After coding, sorting, and identifying the themes, the participants' responses were summarized to each theme and sub-theme. Moreover, some important expressions of the respondents, related to each theme and subtheme, were reported to enrich the research output. In the report of the findings, verbatim quotes have been used to represent each theme and subtheme. The Consolidated Criteria for Reporting Qualitative 


\begin{tabular}{lc}
\hline \multicolumn{1}{l}{ Table 1. Participants characteristics } \\
\hline Participant & Position/Job role \\
\hline $\mathbf{1}$ & Social medicine specialist \\
$\mathbf{2}$ & Faculty member, health services management specialist \\
$\mathbf{3}$ & Head of the national public health management center \\
$\mathbf{4}$ & Social medicine specialist \\
$\mathbf{5}$ & Head of the non-communicable diseases department in provincial health center \\
$\mathbf{7}$ & Human resource manager \\
$\mathbf{8}$ & Head of the health center \\
$\mathbf{9}$ & Faculty member, health education specialist \\
$\mathbf{1 0}$ & Head of health equity research center \\
$\mathbf{1 1}$ & Faculty member, health education specialist \\
$\mathbf{1 3}$ & General director of non-communicable diseases in the ministry of health \\
$\mathbf{1 4}$ & Head of addiction prevention department \\
$\mathbf{1 5}$ & Social medicine specialist \\
$\mathbf{1 6}$ & Head of the national committee for the prevention of non-communicable diseases in the ministry of health \\
\hline
\end{tabular}

Research (COREQ) checklist was used to ensure study rigor, trustworthiness, and transparent reporting. The COREQ checklist contains 32 items broadly categorized into three domains of the research team and reflexivity, study design, as well as data analysis and reporting. The page numbers where each of the items listed in this checklist are reported as an additional file (Supplementary material 1).

\subsection{Ethical considerations}

The study protocol was approved by Tabriz University of Medical Sciences, Tabriz, Iran (IR.TBZMED.REC.1398.887). Participants were informed about the nature of the study, confidentiality, and their right to withdraw at any time without penalty.

\section{Results}

The mean age of the respondents was obtained at 49 years (age range: 43-57 years). Data analysis led to the development of 5 themes and 13 subthemes (Table 2). The derived themes included educational policies, cultural policies, urban transport policies, organizational policies, and economic policies.

\subsection{Theme 1. Educational policies}

4.1.1. Subtheme 1a. Weakness of the educational system in curriculum planning

Respondents agreed that creating unhealthy competition among students, and putting strict regulations in school planning is effective in creating stress among students. In Iran, the most obvious stress-causing issue is the commitment of students to heavy homework loads and exam-oriented system of education, highlighted in high school final exams and Konkoor entrance exam.

According to a participant:

"...Unfortunately, our schools have involved the students in the entrance exam competition. In other words, students are only trying to raise their grades. If we want to improve their happiness, we need to reduce focusing on high grades and entrance exams. The educational curriculum is one of the main causes of stress in our society, actually, the worst possible one, because $98 \%$ of what students learn at high schools is not applicable in academic studies". (P10)

\subsubsection{Subtheme 1b. Failure to learn parenting skills}

According to the participants, parents' lack of awareness about parenting skills harms children's mental health. Ignorance of how to properly deal with children's expectations and desires causes stressful behaviors in them and leads to social harm in adulthood. A faculty member described this problem as follows:

"...The major part of our stress lies in family issues. We do not know how to raise a child. We do not have the required parenting skills. When these children reach the age of 13-14 years, the parents are annoyed and cannot deal with the adolescents. This is highly prevalent in the society". (P8)

\subsubsection{Subtheme 1c. Lack of attention to premarital counseling}

Premarital counseling is one of the most basic measures that can ensure a successful marriage. Positive emotional relationships are a source of happiness; otherwise, family relationships are a great source of stress, suffering, and sorrow. A faculty member noted the lack of attention to learning premarital skills:

"...We have a series of premarital counseling programs; however, many of them are not psychological counseling".

"...Do the couples know that they should have a complete understanding of each other? Many of our stressful tensions are due to the lack of mutual understanding". (P8)

4.2. Theme 2. Cultural policies

4.2.1. Subtheme 2a. Existence of inappropriate custom in society

From the respondents' point of view, happiness is one of the preconditions of a dynamic society. Part 


\begin{tabular}{|c|c|}
\hline Theme & Sub-themes \\
\hline 1. Educational policies & $\begin{array}{l}\text { 1a. Weakness of the educational system in curriculum planning } \\
\text { 1b. Failure to learn parenting skills } \\
\text { 1c. Lack of attention to premarital counseling }\end{array}$ \\
\hline 2. Cultural policies & Existence of inappropriate custom in society \\
\hline 3. Urban transport policies & $\begin{array}{l}\text { 3a. Inappropriate development of transportation network in big cities } \\
\text { 3b. Mismatch of public transportation development and population } \\
\text { 3c. Lack of proper infrastructure for active transportation development }\end{array}$ \\
\hline 4. Organizational policies & $\begin{array}{l}\text { 4a. Inefficiency in developing and enforcing administrative regulations } \\
\text { 4b. Violation of administrative rules }\end{array}$ \\
\hline 5. Economic policies & $\begin{array}{l}\text { 5a. Adverse policies and inefficient management } \\
\text { 5b. Inflation and devaluation of the country's currency } \\
\text { 5c. Insecurity and economic instability } \\
\text { 5d. Mismatch between one's workload and abilities }\end{array}$ \\
\hline
\end{tabular}

of this cheerfulness comes from using happy colors. In Iran, people prefer to use formal colors in choosing their equipment and clothes, which has created a wave of depression in society. A faculty member stated:

"...We live in a society where happiness is a crime, and wearing happy colors is a crime". (P10)

"...The great debate goes back to 'happiness', I do not mean the norms set by people, but the pressure set by the government". (P10)

\subsection{Theme 3. Urban transport policies}

4.3.1. Subtheme 3a. Inappropriate development of transportation network in big cities

According to some participants, the transportation system is one of the most important criteria for well-being in different communities. In this regard, insufficiency or lack of appropriate transportation systems affects the well-being and comfort of the community members. Lack of easy access to buses, taxis, and subways makes people wait a long time to get to their work or any desired destination. Such a long frequent daily waiting to use public transportation increases the levels of stress and anxiety among individuals. A university professor mentioned:

"...Is it really possible to use buses for transportation? It causes you stress. You have to wait for half an hour or an hour. Everyone gathers. When you want to get in, you are pushed and may fight. All of these are caused due to the lack of required infrastructure". (P2)

\subsubsection{Subtheme 3b. Mismatch of public transportation development and population}

Participants acknowledged that congestion in the subway was a problem in major cities. This crowdedness and bustle pressure causes a great deal of stress in people.

"...Sometimes, the subway is too crowded, which is stressful because its capacity is not enough". (P2)

\subsubsection{Subtheme 3c. Lack of proper infrastructure for active transportation development}

Respondents believed that using public transportation required the development of appropriate infrastructure. However, the traffic caused by driving private cars has caused stressful conditions and affected people's mental health. Accidents occurred by non-observance of others' rights also cause more psychological damage to people. In this area, two participants described the current status as the following:

"...There is no infrastructure. If they provide the required infrastructure, no one will use private cars. I am looking for comfort, but the government should create such conditions for me". (P2)

"...We are under stress in traffic. The discussion of 'active transportation' can affect our level of stress. Every day, we should wait too long at red lights, we observe many accidents, and experience much tension and stress". (P8)

\subsection{Theme 4. Organizational policies}

4.4.1. Subtheme 4a. Inefficiency in developing and enforcing administrative regulations

According to the participants, the foundation of an efficient administrative system is formed by correct regulations and simplicity in the implementation process. Failure to comply with the rules and implement the regulations will lead to irregularities in the business licensing process. One manager indicated:

"...Sometimes, the administrative rules and regulations are so cumbersome which, in fact, some of these administrative protocols hinder business growth". (P15)

"...Difficult business conditions can actually lead to stress in people. I work in a private organization; I think that more than $80 \%-90 \%$ of the stress experienced in a private sector is related to the government agencies than the duties or working conditions". (P15)

\subsubsection{Subtheme 4b. Violation of administrative rules}

The compromise of government employees with influential individuals and groups in enforcing laws causes public distrust of the government.

"...Violation of these rules and regulations reduces our social security, which can increase stress and 
distrust in the society". (P15)

\subsection{Theme 5. Economic policies}

Respondents argued that economics played a significant role in influencing people's peace of mind.

\subsubsection{Subtheme 5a. Adverse policies and inefficient management}

The respondents pointed out that the government's weakness in developing formal policy and managing the country is among important sources of the current turmoil in the society.

"...Unfortunately, stressful life that has engulfed the Iranian society is due to poor macro-management of the country". (P8)

\subsubsection{Subtheme 5b. Inflation and devaluation of the country's currency}

Participants noted that inflation and rising prices for basic necessities have worsened people's livelihoods. People are under a lot of psychological pressure to make ends meet.

"...The policies that the country has pursued, such as rising the price of the dollar and inflation, are all stress-causing". (P7)

\subsubsection{Subtheme 5c. Insecurity and economic instability}

Respondents noted that poor economic conditions and economic instability in the society have caused psychological pressure and stress in individuals. A manager described the economic situation of the community as follows:

"...Inflation and economic insecurity are stressful. In order to stop stress, the governments should eliminate economic insecurity at all sectors so that people may relive a little". (P9)

"...This economic insecurity has plagued all social strata, not just the poor. Economic insecurity has put all social classes under stress". (P9)

\subsubsection{Subtheme 5d. A Mismatch between one's workload and abilities}

Based on the responses, as costs increase, people have to work harder to make a living in difficult economic conditions. With an increase in the workload, individuals become stressed and anxious, which affects their personal lives and social relationships. A faculty member stated:

"...Unfortunately, work pressure, economic problems, sanctions, and other factors can significantly increase stress in the society". (P8)

\subsection{Recommendations:}

Participants also mentioned the effective measures in reducing stress (Table 3).

\begin{tabular}{lr}
\hline Table 3. Recommendations & \\
\hline $\mathbf{1}$ & Improving job satisfaction by developing friendly communication skills \\
$\mathbf{2}$ & Creating a happy and refreshing space in the workplace \\
$\mathbf{3}$ & Creating a culture for public physical activity \\
$\mathbf{4}$ & Involving managers and clerics in implementing sports' programs \\
$\mathbf{5}$ & Promoting spirituality \\
$\mathbf{6}$ & Providing adequate facilities and physical space to enhance mental health \\
$\mathbf{7}$ & Improving the community mental health by increasing social vitality \\
$\mathbf{8}$ & Improving the students' mental health by implementing fun programs \\
$\mathbf{9}$ & Creating a culture for developing sports \\
$\mathbf{1 0}$ & Empowering managers in managing the employees' stress \\
$\mathbf{1 2}$ & Having balanced and fair division of duties among employees by the manager \\
$\mathbf{1 3}$ & Recruiting people based on their mental health \\
$\mathbf{1 4}$ & Implementing psychological counseling and social work for employees \\
$\mathbf{1 5}$ & Supporting the unemployed by the cooperation of the Social Security Organization \\
$\mathbf{1 6}$ & Increasing the quality of life by learning and strengthening life skills \\
$\mathbf{1 7}$ & Providing free facilities for attending life skill training classes \\
\hline $\mathbf{1 8}$ & Teaching stress reduction techniques through appropriate exercises \\
$\mathbf{1 9}$ & educational programs for the public \\
\hline $\mathbf{2 0}$ & Improving the education system with the aim of acquiring life skills \\
\hline
\end{tabular}

\section{Discussion}

Based on the findings, a number of different economic, social, political, cultural, and managerial factors play an important role in causing stress. The interaction among these factors hinders the prevention and treatment of high blood pressure. Based on some previous studies, the quality of life is affected by the rate of political, economic, individual, and social developments, as well as values, and customs $(16,17)$. In confirmation of previous studies, stress and anxiety affect the quality of life $(16,18)$ and lead to the development of many diseases, including cardiovascular diseases and hypertension. Lack of studying economic problems and careful economic planning in the country have changed the poverty threshold so that the middle class has fallen into the lower income levels. Economic sanctions, inflation, and costliness have declined incomes and doubled the financial pressures on people (19). In addition, the prevalence of Coronavirus has caused a wide range 
of psychosocial effects at the individual and community levels (20). According to our participants, the high levels of stress caused by prolonged quarantine and the inability to meet living expenses will have a negative impact on health. In such a situation, changing behavioral patterns is not unexpected. In confirmation of our findings, a cross-sectional study in Denmark showed that the residents of disadvantaged neighborhoods had higher levels of stress, compared to the general population. The odds of high-risk behaviors, such as physical inactivity, daily smoking, and lack of fruits or vegetable consumption were also significantly higher in people with low economic status, and stress changed their behavioral patterns (21). Relapse to unhealthy behavior patterns along with other risk factors increases the odds of developing high blood pressure and cardiovascular diseases (22). Moreover, executive problems in organizations and changes in the current laws and regulations were mentioned as other effective stressors. These factors undoubtedly affect the business environment, individuals' productivity and efficiency, as well as the national economic development and progress. In some government agencies, low-level employees abuse their power for personal gain and provide the citizens with certain services and goods after compromisingwith them (23).

In addition to economic and managerial issues, our study findings highlighted incorrect transportation and education policies. In confirmation of other studies, it was found that the wrong and exam-based educational system in Iran has created negative competition among students. Moreover, the existence of education mafia affects the students' viewpoints towards obtaining the best rank in Konkoor, which causes stress in students and harms them greatly (24). The results of a study in Tehran indicated that $81.1 \%$ of the students experienced stress and anxiety about exams, and $59.2 \%$ of these participants reported that this stress was intolerable (25). In addition, a large volume of homework and the use of supplementary textbooks and classes were among the most important factors effective in the students' mental health (24). These findings are also supported by other studies in this area $(26,27)$. According to the participants, poor parenting skills and poor performance in premarital counseling have also caused a great deal of tension and stress in Iran. In confirmation of our findings, other studies indicate that many people cannot solve the problems they face due to lifestyle changes, modernism, and cultural changes (28). Many mental health issues and psychosocial problems are associated with life skills $(29,30)$. Our findings also reveal that urban transport policies affect creating stress. Severe overcrowding to use public transportation not only harms the individuals but also increases their stress levels. Prolonged waiting and daily waste of time lower a person's tolerance threshold resulting in stress, as well as physical and mental damage (31). Due to the lack of proper urban construction for using bicycles and inadequacy of the public transportations, people are forced to use private cars. Traffic jams and accidents caused by failure to obey traffic rules will lead to abnormal behaviors, such as conflict and violence, which affect the individuals' health directly and indirectly (31). Overall, one of the preconditions for social mental health is the provision of a healthy and refreshing environment. A number of studies suggested that feelings of happiness and joy could increase hope and strengthen defenses against stress (32). From the respondents' point of view, factors recommended in this study can influence mental and physical improvement and reduce stress.

\section{Conclusion}

The findings of our study showed that conditions that arise from stress and lack of mental health have stemmed from the government macro-policies and mismanagement. Wrong decisions at the management levels and pressure on people from all walks of life have worsened the situation and harmed people's mental health. Stress, anger, and anxiety in the community have made it difficult to prevent and treat high blood pressure. Lifestyle changes, relapse to unhealthy behavior patterns, and poor quality of life caused by economic and political factors not only complicate the prevention and treatment of hypertension but also are a causative factor of diseases in people. To this end, policymakers are required to change their focus from treatment to social determinants of health and appoint skilled managers to key positions.

\section{Strengths and limitations of the study}

One of the strengths of this study is that it emphasized health in all policies and cross-sectoral cooperation. Given that this study was qualitative in nature, it could not show the problem magnitude well. Therefore, a quantitative study is required over a large population to change the views of Iranian policymakers and managers towards long-term planning with regard to preventing high blood pressure.

\section{Acknowledgments}

The authors would like to appreciate Dr. Jalilian and Dr. Morteza Arab Zozani for their valuable advice. They also wish to thank the participants for their cooperation

\section{Footnotes}

Authors' contributions: $\mathrm{M}-\mathrm{GH}$ obtained the funding. MZ-P contributed to the conception of the research. 
A-J and R-KH-Z designed the methods. FA-B conducted the interviews. FA-B and N-R analyzed and interpreted the data, and FA-B drafted the manuscript. All authors interpreted results and commented on drafts. All authors have read and approved the final version of this manuscript

Ethical Approval: This study was approved by Tabriz University of Medical Sciences, Tabriz, Iran (IR.TBZMED.REC.1398.887). Written informed consent was obtained from all participants in interviews, and consent forms were signed.

Funding/Support: This qualitative study is a part of a Ph.D. dissertation. Funding is provided by the School of Management and Medical Informatics, Tabriz University of Medical Sciences, Tabriz, Iran. The funder had no involvement in the study design, writing the manuscript, or submitting it for publication.

Financial Disclosure: None

Conflicts of Interest: The authors declare that they have no competing interests.

Author note: This qualitative study is part of FA-B's Ph.D. dissertation.

Availability of data and materials: The transcripts/data of this qualitative study are not publicly available due to confidentiality agreements with the participants.

\section{References}

1. Awuah RB, de-Graft Aikins A, Dodoo FN, Meeks KA, Beune EJ, Klipstein-Grobusch $\mathrm{K}$, et al. Psychosocial factors and hypertension prevalence among Ghanaians in Ghana and Ghanaian migrants in Europe: The RODAM study. Health Psychol Open. 2019;6(2):2055102919885752. doi: 10.1177/2055102919885752. [PubMed: 31763049].

2. Jongen VW, Lalla-Edward ST, Vos AG, Godijk NG, Tempelman $\mathrm{H}$, Grobbee DE, et al. Hypertension in a rural community in South Africa: what they know, what they think they know and what they recommend. BMC Public Health. 2019;19(1):341. doi: 10.1186/s12889-019-6642-3. [PubMed: 30909905].

3. Hu B, Liu X, Yin S, Fan H, Feng F, Yuan J. Effects of psychological stress on hypertension in middle-aged Chinese: a crosssectional study. PLoS One. 2015;10(6):e0129163. doi: 10.1371/journal.pone.0129163. [PubMed: 26043027].

4. Carey RM, Muntner P, Bosworth HB, Whelton PK. Reprint of: prevention and control of hypertension: JACC Health Promotion Series. J Am Coll Cardiol. 2018;72(23):2996-3011. doi: 10.1016/j.jacc.2018.10.022. [PubMed: 30522633].

5. Havranek EP, Mujahid MS, Barr DA, Blair IV, Cohen MS, CruzFlores S, et al. Social Determinants of Risk and Outcomes for Cardiovascular Disease: A Scientific Statement From the American Heart Association. Circulation. 2015;132(9):873-98. doi: 10.1161/CIR.0000000000000228. [PubMed: 26240271].

6. Daniel H, Bornstein SS, Kane GC. Addressing social determinants to improve patient care and promote health equity: an American College of Physicians Position Paper. Ann Intern Med. 2018;168(8):577-8. doi: 10.7326/M17-2441. [PubMed: 29677265].

7. Liu MY, Li N, Li WA, Khan H. Association between psychosocial stress and hypertension: a systematic review and meta-analysis. Neurol Res. 2017;39(6):573-80. doi: 10.1080/01616412.2017.1317904. [PubMed: 28415916].

8. Amiri P, Vahedi-Notash G, Naseri P, Khalili D, Nazari SS, Mehrabi Y, et al. National trends of pre-hypertension and hypertension among Iranian adolescents across urban and rural areas (2007-2011). Biol Sex Differ. 2019;10(1):15. doi: 10.1186/s13293-019-0230-1. [PubMed: 30922399].

9. Lu X, Juon HS, He X, Dallal CM, Wang MQ, Lee S. The association between perceived stress and hypertension among asian americans: does social support and social network make a difference? J Community Health. 2019;44(3):451-62. doi: 10.1007/s10900-018-00612-7. [PubMed: 30604222].

10. Tabrizi JS, Sadeghi-Bazargani H, Farahbakhsh M, Nikniaz L, Nikniaz Z. Prevalence and associated factors of prehypertension and hypertension in Iranian population: the lifestyle promotion project (LPP). PloS One. 2016;11(10): e0165264. doi: 10.1371/journal.pone.0165264. [PubMed: 27783691]

11. Mohsenzadeh Y, Motedayen M, Hemmati F, Sayehmiri K, Sarokhani MT, Sarokhani D. Investigating the prevalence rate of hypertension in Iranian men and women: a study of systematic review and meta-analysis. J Basic Res Med Sci. 2017;4(1):53-62. doi: 10.18869/acadpub.jbrms.4.1.53.

12. Sadeghi M, Haghdoost AA, Bahrampour A, Dehghani M. Modeling the burden of cardiovascular diseases in Iran from 2005 to 2025: The impact of demographic changes. Iran J Public Health. 2017;46(4):506-16. [PubMed: 28540267].

13. Tan ST, Quek RY, Haldane V, Koh JJ, Han EK, Ong SE, et al. The social determinants of chronic disease management: perspectives of elderly patients with hypertension from low socio-economic background in Singapore. Int J Equity Health. 2019;18(1):1. doi: 10.1186/s12939-018-0897-7. [PubMed: 30606218].

14. Cuspidi C, Tadic M, Grassi G, Mancia G. Treatment of hypertension: the ESH/ESC guidelines recommendations. Pharmacol Res. 2018;128:315-21. doi: 10.1016/j.phrs.2017.10.003. [PubMed: 29080798].

15. Saunders B, Sim J, Kingstone T, Baker S, Waterfield J, Bartlam $B$, et al. Saturation in qualitative research: exploring its conceptualization and operationalization. Qual Quant. 2018; 52(4):1893-907. doi: 10.1007/s11135-017-0574-8. [PubMed: 29937585].

16. Lee JE, Kim YJ, Park HJ, Park S, Kim H, Kwon O. Association of recommended food score with depression, anxiety, and quality of life in Korean adults: the 2014-2015 National Fitness Award Project. BMC Public Health. 2019;19(1):956. doi: 10.1186/s12889-019-7298-8. [PubMed: 31315605].

17. Jung ES, Choi SK, Baek YS. Factors influencing the quality of life of community residents. J Digital Converg. 2015;13(11):231-9. doi: 10.14400/JDC.2015.13.11.231.

18. Sivertsen H, Bjørkløf GH, Engedal K, Selbæk G, Helvik AS. Depression and quality of life in older persons: a review. Dement Geriatr Cogn Disord. 2015;40(5-6):311-39. doi: 10.1159/000437299. [PubMed: 26360014].

19. Aloosh M, Salavati A, Aloosh A. Economic sanctions threaten population health: the case of Iran. Public Health. 2019;169:10-3. doi: 10.1016/j.puhe.2019.01.006. [PubMed: 30771721].

20. Mattioli AV, Nasi M, Cocchi C, Farinetti A. COVID-19 outbreak: impact of the quarantine-induced stress on cardiovascular disease risk burden. Future Med. 2020;16(6):539-42. doi: 10.2217/fca-2020-0055. [PubMed: 32351128].

21. Algren MH, Ekholm O, Nielsen L, Ersbøll AK, Bak CK, Andersen PT. Associations between perceived stress, socioeconomic status, and health-risk behaviour in deprived neighbourhoods in Denmark: a cross-sectional study. BMC Public Health. 2018;18(1):250. doi: 10.1186/s12889-018-5170-x. [PubMed: 29439681].

22. Espejo M, Magabo S, Rivera-Castro A, Faiz M, Ramirez L, Robles $\mathrm{C}$, et al. Qualitative Study of knowledge, perception, and behavior related to hypertension and cardiovascular disease risk reduction among hypertensive African-Americans in urban inner city of South Bronx, New York. J Racial Ethn Health Disparities. 2019;6(1):197-206. doi: 10.1007/s40615-0180514-x. [PubMed: 30117092].

23. Al-Jundi SA, Shuhaiber A, Al-Emara SS. The effect of culture and organisational culture on administrative corruption. Int J Econ Bus Res. 2019;18(4):436-51. doi: 10.1504/IJEBR.2019.103096. 
24. Hosseinkhani Z, Nedjat S, Hassanabadi HR, Parsaeian M. Academic stress from the viewpoint of Iranian adolescents: a qualitative study. J Educ Health Promot. 2019;8:13. doi: 10.4103/jehp.jehp_202_18. [PubMed: 30815484].

25. Noury R, Kelishadi R, Ziaoddini H. Study of common stresses among students in Tehran. J Isfahan Med Sch. 2010;28(105): $1-12$.

26. Sung YT, Chao TY, Tseng FL. Reexamining the relationship between test anxiety and learning achievement: an individualdifferences perspective. Contemp Educ Psychol. 2016;46:24152. doi: 10.1016/j.cedpsych.2016.07.001.

27. Khanehkeshi A. A comparative study of the academic stress and depression among high school girl and boy students. $J$ Educ Psychol. 2012;6(1):11-20. doi: 10.26634/JPSY.6.1.1878.

28. Jafarigiv S, Peyman N. The effect of life skills training with health literacy strategies on self-esteem and self-efficacy in female students during puberty. Int J Adolesc Med Health. 2019;20:121. doi: 10.1515/ijamh-2019-0121. [PubMed: 31539358].
29. Jalali F, Hashemi SF. Group life skills training for promoting mental health in women after released from prison: a randomized pilot efficacy trial. Health Care Women Int. 2019;40(3):314-27. doi: 10.1080/07399332.2018.1547385. [PubMed: 30860958].

30. Naeim M, Rahimi HR, Soltani F, Farazandeh F, Nejad FM, Sharafi MR, et al. The role of life skills and self-efficacy in mental health among male students in High school. Int J Med Res Health Sci. 2016;5(7S):118-26.

31. Nadrian H, Taghdisi MH, Aghemiri M, Khazaee-Pool $M$, Shojaeizadeh D. Impacts of urban traffic jams on physical health of residents from the inhabitants 'perspective in Sanandaj city (a qualitative study). Iran $J$ Health Educ Health Promot. 2017;5(4):345-58. doi: 10.30699/acadpub.ijhehp.5.4.345.

32. Moeini B, Barati M, Farhadian M, Ara MH. The association between social support and happiness among elderly in Iran. Korean J Fam Med. 2018;39(4):260-5. doi: 10.4082/kjfm.17.0121. [PubMed: 29972894].

The Consolidated Criteria for Reporting Qualitative Research Checklist: A 32-item checklist for interviews and focus groups of participants.

\begin{tabular}{|c|c|c|c|}
\hline Topic & $\begin{array}{c}\text { Item } \\
\text { No. }\end{array}$ & Guiding Questions/Description & $\begin{array}{c}\text { Reported on } \\
\text { page no. }\end{array}$ \\
\hline \multicolumn{4}{|l|}{$\begin{array}{l}\text { Domain 1: Research team and } \\
\text { reflexivity }\end{array}$} \\
\hline \multicolumn{4}{|l|}{ Personal characteristics } \\
\hline Interviewer/facilitator & 1 & Which author/s conducted the interview or focus group? & 6 \\
\hline Credentials & 2 & What were the researcher's credentials? e.g., Ph.D., MD. & 19 \\
\hline Occupation & 3 & What was their occupation at the time of the study? & 19 \\
\hline Gender & 4 & Was the researcher male or female? & $18 \& 19$ \\
\hline Experience and training & 5 & What experience or training did the researcher have? & 19 \\
\hline \multicolumn{4}{|l|}{ Relationship with participants } \\
\hline Relationship established & 6 & $\begin{array}{l}\text { Was a relationship established prior to study } \\
\text { commencement? }\end{array}$ & 5 \\
\hline $\begin{array}{l}\text { Participant knowledge of the } \\
\text { interviewer }\end{array}$ & 7 & $\begin{array}{l}\text { What did the participants know about the researcher? e.g., } \\
\text { personal goals, reasons for doing the research }\end{array}$ & 5 \\
\hline Interviewer characteristics & 8 & $\begin{array}{c}\text { What characteristics were reported about the inter } \\
\text { viewer/facilitator? e.g., bias, assumptions, reasons, and } \\
\text { interests in the research topic }\end{array}$ & NO \\
\hline \multirow{2}{*}{\multicolumn{4}{|c|}{$\begin{array}{l}\text { Domain 2: Study design } \\
\text { Theoretical framework }\end{array}$}} \\
\hline & & & \\
\hline $\begin{array}{l}\text { Methodological orientation and } \\
\text { Theory }\end{array}$ & 9 & $\begin{array}{l}\text { What methodological orientation was stated to underpin } \\
\text { the study? e.g., grounded theory, discourse analysis, } \\
\text { ethnography, phenomenology, content analysis }\end{array}$ & 6 \\
\hline \multicolumn{4}{|l|}{ Participant selection } \\
\hline Sampling & 10 & $\begin{array}{l}\text { How were the participants selected? e.g., purposive, } \\
\text { convenience, consecutive, snowball }\end{array}$ & 5 \\
\hline Method of approach & 11 & $\begin{array}{l}\text { How were the participants approached? e.g., face-to-face, } \\
\text { telephone, mail, email }\end{array}$ & 5 \\
\hline Sample size & 12 & How many participants were in the study? & 5 \\
\hline Non-participation & 13 & $\begin{array}{l}\text { How many people refused to participate or dropped out? } \\
\text { Reasons? }\end{array}$ & NO \\
\hline \multicolumn{4}{|c|}{ 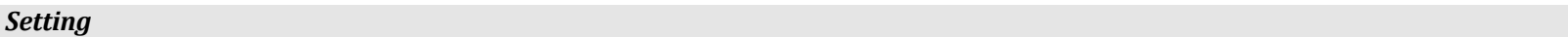 } \\
\hline Setting of data collection & 14 & Where was the data collected? e.g., home, clinic, workplace & 6 \\
\hline Presence of non-participants & 15 & $\begin{array}{l}\text { Was anyone else present besides the participants and } \\
\text { researchers? }\end{array}$ & NO \\
\hline Description of sample & 16 & $\begin{array}{l}\text { What are the important characteristics of the sample? e.g., } \\
\text { demographic characteristics, date }\end{array}$ & 6 \\
\hline
\end{tabular}


The consolidated criteria for reporting qualitative research checklist: a 32-item checklist for interviews and focus groups of participants (continued)

\begin{tabular}{|c|c|c|c|}
\hline Topic & $\begin{array}{l}\text { Item } \\
\text { No. }\end{array}$ & Guide Questions/Description & $\begin{array}{l}\text { Reported on } \\
\text { page no. }\end{array}$ \\
\hline \multicolumn{4}{|l|}{ Data collection } \\
\hline Interview guide & 17 & $\begin{array}{l}\text { Were questions, prompts, guides provided by the authors? Was it pilot } \\
\text { tested? }\end{array}$ & $\begin{array}{l}6 \text { Additional } \\
\text { file } 1\end{array}$ \\
\hline Repeat interviews & 18 & Were repeats interviews carried out? If yes, how many? & NO \\
\hline Audio/visual recording & 19 & Did the research use audio or visual recording to collect the data? & 6 \\
\hline Field notes & 20 & $\begin{array}{l}\text { Were field notes made during and/or after the interview or focus } \\
\text { group? }\end{array}$ & NO \\
\hline Duration & 21 & What was the duration of the interviews or focus group? & 5 \\
\hline Data saturation & 22 & Was data saturation discussed? & 6 \\
\hline Transcripts returned & 23 & $\begin{array}{l}\text { Were transcripts returned to participants for comment and/or } \\
\text { correction? }\end{array}$ & NO \\
\hline \multicolumn{4}{|l|}{ Domain 3: Analysis and findings } \\
\hline \multicolumn{4}{|l|}{ Data analysis } \\
\hline Number of data coders & 24 & How many data coders coded the data? & 6 \\
\hline Description of the coding tree & 25 & Did authors provide a description of the coding tree? & 6 \\
\hline Derivation of themes & 26 & Were themes identified in advance or derived from the data? & 6 Table 2 \\
\hline Software & 27 & What software, if applicable, was used to manage the data? & 6 \\
\hline Participant checking & 28 & Did participants provide feedback on the findings? & NO \\
\hline \multicolumn{4}{|l|}{ Reporting } \\
\hline Quotations presented & 29 & $\begin{array}{l}\text { Were participant quotations presented to illustrate the } \\
\text { themes/findings? Was each quotation identified? e.g., participant } \\
\text { number }\end{array}$ & $7-11$ \\
\hline Data and findings consistency & 30 & Was there consistency between the data presented and the findings? & $11-14$ \\
\hline Clarity of major themes & 31 & Were major themes clearly presented in the findings? & $11-14$ \\
\hline Clarity of minor themes & 32 & Is there a description of diverse cases or discussion of minor themes? & NO \\
\hline
\end{tabular}

